\title{
Mild evolution of the stellar metallicity gradients of disc galaxies
}

\author{
Patricia B. Tissera ${ }^{1,2}$, Rubens E. G. Machado ${ }^{1,3,4}$ José M. Vilchez $^{5}$, Susana E. Pedrosa ${ }^{6}$, \\ Patricia Sanchez-Blazquez ${ }^{7,8}$, and Silvio Varela ${ }^{1}$
}

\author{
1 Departamento de Ciencias Fisicas, Universidad Andres Bello, Fernandez Concha 700, Santiago, Chile \\ e-mail: patricia.tissera@unab.cl \\ 2 Millennium Institute of Astrophysics, Av. Republica 220, Santiago, Chile \\ 3 Universidade Federal de Ouro Preto, Departamento de Física, Campus Universitario Morro do Cruzeiro, 35400-000 Ouro Preto, \\ Brazil \\ 4 Departamento Academico de Física, Universidade Tecnológica Federal do Paraná, Rua Sete de Setembro 3165, 80230-901 Curitiba, \\ Brazil \\ 5 Instituto de Astrofísica de Andalucía (CSIC), Glorieta de la Astronomía s/n, 18008 Granada, Spain \\ 6 Instituto de Astronomía y Física del Espacio, CONICET-UBA, Casilla de Correos 67, Suc. 28, C1428ZAA, \\ Ciudad Autónoma de Buenos Aires, Argentina \\ 7 Departamento de Física Teórica, Universidad Autónoma de Madrid, 28049 Madrid, Spain \\ 8 Instituto de Astrofísica, Universidad Pontifica Católica de Chile, Av. Vicuña Mackenna 4860, Santiago, Chile
}

Received 12 May 2016 / Accepted 8 April 2017

\begin{abstract}
Context. The metallicity gradients of the stellar populations in disc galaxies and their evolution store relevant information on the disc formation history and on those processes which could mix stars a posteriori, such as migration, bars and/or galaxy-galaxy interactions. Aims. We aim to investigate the evolution of the metallicity gradients of the whole stellar populations in disc components of simulated galaxies in a cosmological context.

Methods. We analyse simulated disc galaxies selected from a cosmological hydrodynamical simulation that includes chemical evolution and a physically motivated supernova feedback capable of driving mass-loaded galactic winds.

Results. We detect a mild evolution with redshift in the metallicity slopes of $-0.02 \pm 0.01 \mathrm{dex} \mathrm{kpc}^{-1}$ from $z \sim 1$. If the metallicity profiles are normalised by the effective radius of the stellar disc, the slopes show no clear evolution for $z<1$, with a median value of approximately $-0.23 \mathrm{dex} r_{\mathrm{eff}}^{-1}$. As a function of stellar mass, we find that metallicity gradients steepen for stellar masses smaller than $\sim 10^{10.3} M_{\odot}$ while the trend reverses for higher stellar masses, in the redshift range $z=[0,1]$. Galaxies with small stellar masses have discs with larger $r_{\text {eff }}$ and flatter metallicity gradients than expected. We detect migration albeit weaker than in previous works.

Conclusions. Our stellar discs show a mild evolution of the stellar metallicity slopes up to $z \sim 1$, which is well-matched by the evolution calculated archeologically from the abundance distributions of mono-age stellar populations at $z \sim 0$. The dispersion in the relations allows for stronger individual evolutions. Overall, supernova feedback could explain the trends but an impact of migration can not be totally discarded. Galaxy-galaxy interactions or small satellite accretions can also contribute to modify the metallicity profiles in the outer parts. Disentangling the effects of these processes for individual galaxies is still a challenge in a cosmological context.
\end{abstract}

Key words. galaxies: abundances - galaxies: formation - galaxies: evolution - galaxies: ISM

\section{Introduction}

The chemical abundances of the stellar populations (SPs) in galaxies of different morphologies provide important clues to understand their history of formation. Chemical patterns store relevant information on the properties of the interstellar medium (ISM) and on the physical processes that might modify them along the galaxy evolutionary paths (e.g. Matteucci \& Greggio 1986; Mollá et al. 1997; Chiappini et al. 2001; Pipino et al. 2008; Gibson et al. 2013; Tissera et al. 2016b). In an insideout scenario for disc formation, gas-phase metallicity profiles with negative gradients are a natural outcome if star formation is driven mainly by the gas density in the discs. Supernova (SN) feedback can regulate the star formation activity and modulate the metallicity content of galaxies if mass-loaded galactic winds are triggered (Gibson et al. 2013; Tissera et al. 2016b). New-born stars acquire the chemical abundances of the ISM from where they formed. Later on, the stellar abundance distributions could be affected by dynamical processes that redistribute stars in the disc components such as bars, migration (e.g. Minchev et al. 2014), or galaxy-galaxy interactions and mergers (e.g. Di Matteo et al. 2009; Perez et al. 2011).

In the Local Universe, most of our current knowledge of chemical distributions in disc galaxies comes from studies of the ISM via observations of HII regions or young stellar populations. Current observations are consistent with the metallicity profiles of the ISM having negative metallicity gradients, on average. Metallicity gradients in units of dex kpc${ }^{-1}$ determine tight correlations with the global properties of galaxies such as the stellar mass or the size, which are erased when normalised by a characteristics radius such as the half-mass radius (e.g. Zaritsky et al. 1994; Sánchez et al. 2013). There are few indirect estimations of the evolution of the stellar metallicity gradients using planetary nebulae (PN; e.g. Henry et al. 2010; Stanghellini et al. 2010). Maciel et al. (2003) calculated the gradients of SPs with 
different ages in the Milky Way disc, finding a signal of increasingly negative metallicity gradients for older stars. Recently Magrini et al. (2016) analysed the metallicity gradients determined by PN for four nearby galaxies, finding them to be flatter than those detected using HII regions. Surveys such as CALIFA provide detailed information on the properties of the ISM and the SPs, including their chemical abundances and age distributions on a variety of galaxies (Sánchez-Blázquez et al. 2014; González Delgado et al. 2015). The SDDS-IV MaNGA survey (Bundy et al. 2015) also investigate spatially resolved SPs and radial age and metallicity gradients for nearby galaxies ( $\mathrm{Li}$ et al. 2015; Wilkinson et al. 2015) and will provide a large statistical sample to confront with models. The available observations of HII regions for high- $z$ galaxies do not allow the formation of a robust conclusion on the evolution of the metallicity gradients (e.g. Yuan et al. 2011; Queyrel et al. 2012; Stott et al. 2014; Jones et al. 2015). In fact, high-redshift observations show a complex situation with gas-phase components showing a variety of metallicity gradients that could respond to the action of different physical processes (e.g. Cresci et al. 2010).

A large effort has been invested in understanding the chemical patterns of galaxies using analytical and numerical chemical modelling (e.g. Brook et al. 2007; Calura et al. 2012; Mollá et al. 2015). In particular, hydrodynamical simulations provide the chemical enrichment of baryons as galaxies are assembled in a cosmological framework, opening the possibility of understanding the interplay of different physical processes in the non-linear regime of evolution (e.g. Mosconi et al. 2001; Lia et al. 2002; Wiersma et al. 2009). Pilkington et al. (2012) carried out a comparison of the metallicity gradients in the ISM (traced by young SPs) obtained by different numerical and analytical models. Gibson et al. (2013) analysed different models of SN feedback schemes reporting different evolution for the gas-phase metallicity gradients. No evolution was reported when an enhanced SN model was used. Tissera et al. (2016b) studied the gas-phase metallicity gradients in discs and the specific star formation of the galaxies. For gas-phase metallicities, the simulated galaxies showed a correlation with stellar mass, which was erased when the metallicity gradients were renormalised by the effective radius, reproducing observations by Ho et al. (2015, and references there in). They also found indications of a correlation between the abundance slopes and the specific star formation rate (sSFR) in agreement with observational findings (Stott et al. 2014). As a function of redshift, the metallicity gradients of the gas-phase disc components were found to be more negative at higher redshift, principally for lower stellar-mass galaxies. The fraction of galaxies with positive metallicity gradients increased with increasing redshift and were found to be associated to mergers and interactions. The trends found for the gas-phase metallicity gradients by Tissera et al. (2016b) are relevant to the discussion of this paper because we are using the same set of simulated galaxies.

The evolution of the metallicity gradients of the SPs provides important and complementary information on the chemodynamical evolution of galaxies. This is not only determined by the formation of discs but also by those physical mechanisms that can disturb gradients a posteriori, such as bar formation, migration, and galaxy interactions. Galaxy interactions are known observationally (Lambas et al. 2003; Ellison et al. 2008; Rupke et al. 2010; Michel-Dansac et al. 2008) and numerically (Barnes \& Hernquist 1996; Tissera 2000; Perez et al. 2006, 2011) to be an efficient mechanism to trigger gas inflows that transport low-metallicity material to inner regions. As a consequence, the gas-phase metallicity profiles become flatter.
These gas inflows can trigger significant starbursts, which produce a two-fold effect: the new-ejected enriched material can enrich both the central regions, and steepen the metallicity gradients, and the outer parts of the galaxies via galactic fountains (Perez et al. 2011; Di Matteo et al. 2013). The new-born stars might reflect these changes in metallicity depending on the relative contribution of the different stellar populations (Perez et al. 2011). Migration can also contribute to flatten the stellar chemical profiles by mixing them and transporting old stars to the outer regions. This process is likely to be related to the transient spiral arms (Sellwood \& Binney 2002) or the effects of galaxy interactions, which might produce radial mixing (Quillen et al. 2009). Recent results using high-resolution simulations reported a more clear detection of migration, which showed the displacement of old stars towards the outer part of the discs when a bar structure formed (Grand et al. 2016). It is important to note that if galaxies form in a hierarchical clustering scenario, there are several processes that can affect the mixing of chemical elements in the ISM from which stars are formed (e.g. gas inflows, gas outflows, mergers), and later on, the distribution of the stellar populations (e.g. minor mergers, migration, bar formation). Disentangling their effects is still a complicate task.

In this paper, we study the evolution of the metallicity gradients of the SPs in the disc components of the set of simulated galaxies analysed by Tissera et al. $(2016 b, a)$ at $z \sim 0$. These authors reported the normalised and non-normalised stellar metallicity gradients to be in agreement with results obtained for galaxies in the CALIFA survey by Sánchez-Blázquez et al. (2014). At $z \sim 0$, the simulated stellar discs were reported to have half-mass radii $\left(r_{\text {eff }}\right)$ in very good agreement with observations (Sánchez-Blázquez et al. 2014; van der Wel et al. 2014). This is an important outcome of our models considering that the free parameters were not fine-tuned to achieve this trend. Overall, the simulated disc galaxies formed mainly in an inside-out fashion, from gas which conserved its specific angular momentum content. And if the discs are not perturbed, negative metallicity profiles are naturally formed as expected.

At $z \sim 0$, Tissera et al. (2016a) analysed the metallicity profiles of the stellar populations of the same discs we use in this work and reported a change in the slope of the relation between metallicity gradients and stellar mass, resembling a U-shape. These authors point out that this feature is more significant in the old SPs (i.e. older than $6 \mathrm{Gyr}$ ) than the young stars (i.e. younger than 2 Gyr). Old stars are reported to have slightly steeper metallicity gradients than young SPs, on average. A similar trend can be seen in CALIFA's results from Sánchez-Blázquez et al. (2014) and González Delgado et al. (2015). In this paper, we extend the analysis done by Tissera et al. (2016a) to $z \sim 1$. We note that our galaxy sample provides different histories of formation for each system in agreement with the current cosmological framework, for the same sub-grid physics.

This paper is organised as follows. Section 2 summarises the characteristics of the simulation and the galaxy catalogue. Section 3 describes the analysis and Conclusions summarises the main results.

\section{Simulated galaxies}

We analysed the same set of disc galaxies studied by Tissera et al. (2016a,b). The details on the numerical code used and the determination of the properties of discs can be found in the mentioned works and in Pedrosa \& Tissera (2015). Here we summarise the main aspects. The analysed galaxies were selected from a cosmological volume consistent with the $\Lambda$ Cold 
Dark Matter scenario (the so-called simulation S230D from the Fenix Project) with $\Omega_{\Lambda}=0.7, \Omega_{\mathrm{m}}=0.3, \Omega_{\mathrm{b}}=0.04$, a normalisation of the power spectrum of $\sigma_{8}=0.9$ and $H_{0}=$ $100 h \mathrm{~km} \mathrm{~s}^{-1} \mathrm{Mpc}^{-1}$, with $h=0.7$. The simulated volume represents a box of $14 \mathrm{Mpc}$ comoving side, resolved with $2 \times 230^{3}$ initial particles, achieving a mass resolution of $5.9 \times 10^{6} \mathrm{~h}^{-1} M_{\odot}$ and $9.1 \times 10^{5} h^{-1} M_{\odot}$ for the dark matter and initial gas particles, respectively. The gravitational softening is $0.7 \mathrm{kpc}$.

We apply a version of the code GADGET-3 (Springel 2005), optimised for massive parallel simulations of highly inhomogeneous systems. This version includes treatments for metaldependent radiative cooling, stochastic star formation (SF), chemical enrichment, and a multiphase model for the ISM and the supernova (SN) feedback scheme of Scannapieco et al. (2006). The SN feedback model was able to successfully trigger galactic mass-loaded winds without introducing mass-scale free parameters. The code considers energy feedback by Type II (SNII) and Type Ia (SNIa) supernovae. The code also includes the chemical evolution model developed by Mosconi et al. (2001). SNII and SNIa contributed with chemical elements estimated by adopting the chemical yields of Woosley \& Weaver (1995) and Iwamoto et al. (1999), respectively.

We use the galaxy catalogue constructed by Tissera et al. (2016b). The spheroidal and disc components are separated by applying a dynamical criterium based on the angular momentum content and the binding energy momentum component in the direction of the total angular momentum, and $J_{z, \max }(E)$ is the maximum $J_{z}$ over all particles at a given binding energy $E$ (see Tissera et al. 2012, for details on the procedure and conditions used). At all analysed redshifts, only discs defined with more than 2000 star particles are studied. A few systems were discarded when the morphology did not allow for a robust linear fit to the metallicity profile, as in galaxies strongly perturbed by close interactions, for example. After the removal of such objects, the adopted resolution criterium yielded 37, 23, 20 and 17 galaxies, for redshifts $z \sim 0,0.4,0.95$ and 1.3 , respectively. We note that the number of discs in the samples decreased for increasing redshift since we are maintaining the requirement of 2000 star particles to select the systems to be analysed for all redshifts.

\section{Analysis}

Before analysing the evolution of metallicity profiles, it is important to discuss if the sizes of the simulated galaxies evolve as a function of redshift as expected from observations. At $z \sim 0$, Tissera et al. (2016a) reported the $r_{\text {eff }}$ of the stellar discs to be in good agreement with observations. Here, we assess to what extent our simulated $r_{\text {eff }}$ are consistent with observations as a function of redshift. Figure 1 shows the simulated stellar $r_{\text {eff }}$ as a function of total stellar mass in the four analysed redshift intervals and confronts them with observational estimations by van der Wel et al. (2014). As can be seen from this figure, the simulated galaxies have comparable $r_{\text {eff }}$, which evolves similarly to the observed values estimated for late-type galaxies. We note that observations used the light-weighted distributions to estimate the effective radius while we are considering the stellar mass distributions. For this comparison, the $r_{\mathrm{eff}}$ are estimated using all stars in the main galaxies. At a given stellar mass, there is a significant dispersion as can be appreciated from this figure, which is compatible with the observed values reported by van der Wel et al. (2014). Overall, we find that the decrease in size as a function of stellar mass with redshift is well-reproduced by our simulated discs in the analysed redshift range.

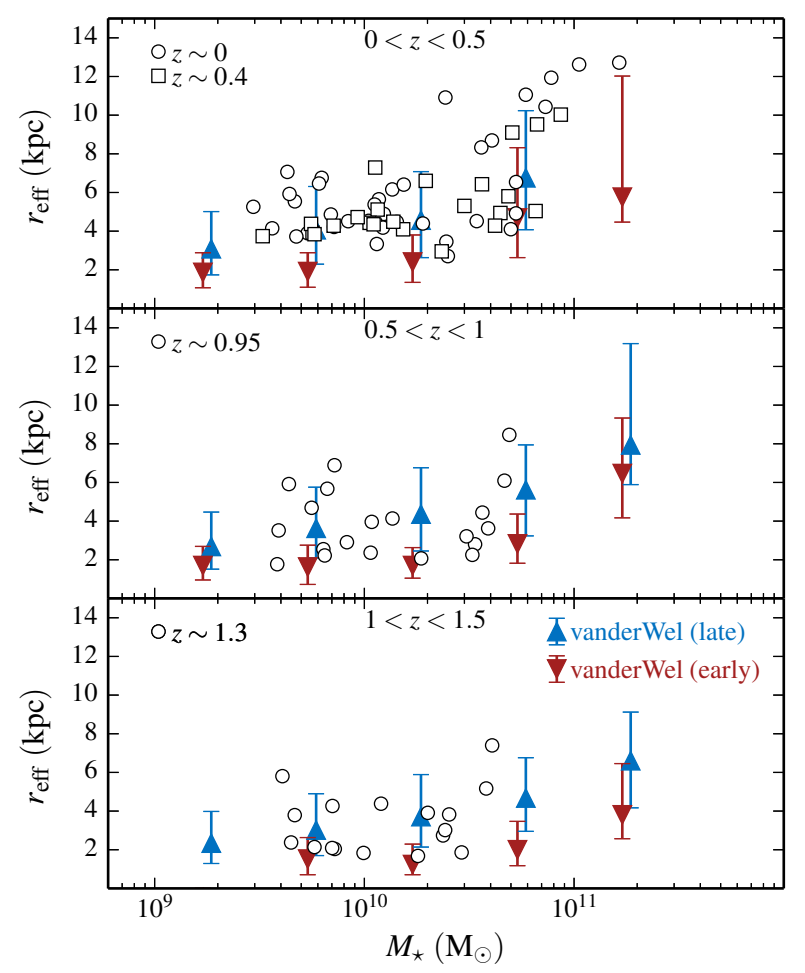

Fig. 1. Mean effective stellar radii for all simulated galaxies (open symbols) as a function of stellar mass for the four analysed redshifts. The observational data points of van der Wel et al. (2014) in three redshift intervals are included for comparison and slightly offset horizontally for clarity.

The disc sizes are determined by the angular momentum content of the infalling gas but they can also be affected by SN feedback and dynamical processes such as mergers secular evolution and/or migration. Pedrosa \& Tissera (2015) found that galaxies in our sample have stellar and gaseous discs formed consistently with the standard disc scenario based on angular momentum conservation (Fall \& Efstathiou 1980). These simulated galaxies reproduce observed trends between the specific angular momentum content, the potential well and the morphology (Romanowsky \& Fall 2012), and these relations are conserved as a function of redshift in this simulation. In the analysed redshift range, Pedrosa et al. (2014) reported these galaxies to have not experienced major mergers since $z \sim 3$, which could have significantly disturbed the angular momentum content and the size of the systems. Hence, for the analysed galaxies, the main path of disc formation is the infall of gas with specific angular conservation. Then the gas is transformed into stars in the discs. Afterwards, other mechanisms such as migration, galaxy mergers, or interactions, can play a role in mixing stellar populations of different ages and chemical content (e.g. Grand et al. 2016).

\subsection{Metallicity profiles of the stellar populations}

We estimate the radial abundance profiles of the SPs in the selected simulated discs for the four mentioned redshifts. We perform linear regression fits by applying a bootstrap technique to the metallicity profiles. In Fig. 2, we show the stellar metallicity gradients as a function of stellar mass for the four analysed redshifts (upper panels). In the lower panels of this figure, the renormalised systems are displayed. As the minimum number of stellar particles are kept fixed as a function of redshift, the 


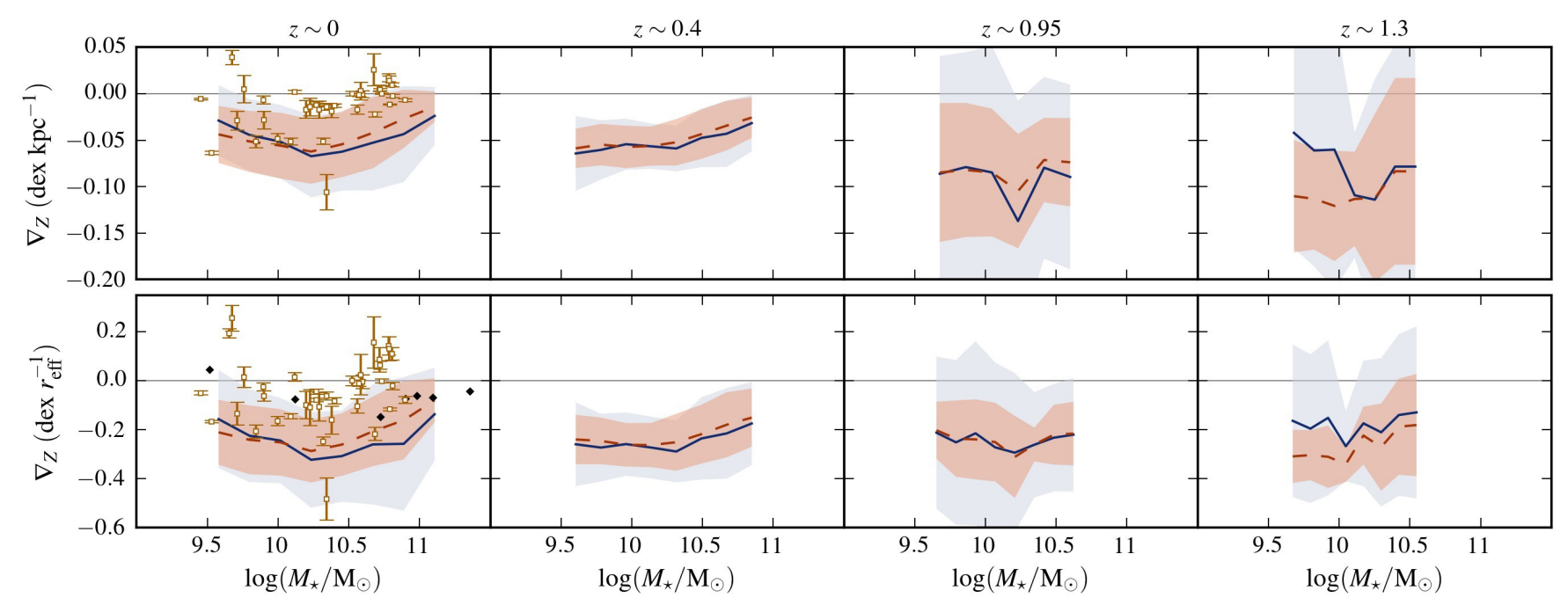

Fig. 2. Metallicity gradients as a function of stellar mass for the analysed disc galaxies at $z \sim 0$ (upper panels). The normalised gradients are displayed in the lower panels. Observational results for the metallicity gradients of the stellar discs in the CALIFA survey by Sánchez-Blázquez et al. (2014; black diamonds) and González Delgado et al. (2015; yellow crosses, lower panel) are also displayed at $z \sim 0$ for comparison. Mean estimations and their standard dispersion in both radial intervals: $[0.5,1.5] r_{\mathrm{eff}}$ (blue shades and lines) and $[0.5,1.0] r_{\mathrm{eff}}$ (pink shades and lines) are included for comparison.

Table 1. Median stellar metallicity gradients in dex $\mathrm{kpc}^{-1}$ and dex $r_{\mathrm{eff}}^{-1}$ for all, low and high stellar-mass galaxies in the $z=[0,1.3]$ interval.

\begin{tabular}{|c|c|c|c|c|c|}
\hline \multirow[b]{2}{*}{$z$} & \multirow[b]{2}{*}{$\begin{array}{l}M_{\star} \\
M_{\odot} \\
\end{array}$} & \multicolumn{2}{|c|}{$\left[0.5 r_{\mathrm{eff}}: 1.0 r_{\mathrm{eff}}\right]$} & \multicolumn{2}{|c|}{$\left[0.5 r_{\mathrm{eff}}: 1.5 r_{\mathrm{eff}}\right]$} \\
\hline & & $\begin{array}{c}\nabla_{Z} \\
\left(\operatorname{dex~kpc}{ }^{-1}\right)\end{array}$ & $\begin{array}{c}\nabla_{Z} \\
\left(\operatorname{dex} r_{\text {eff }}^{-1}\right)\end{array}$ & $\begin{array}{c}\nabla_{Z} \\
\left(\operatorname{dex~kpc}{ }^{-1}\right) \\
\end{array}$ & $\begin{array}{c}\nabla_{Z} \\
\left(\operatorname{dex} r_{\text {eff }}^{-1}\right) \\
\end{array}$ \\
\hline$z=0.0$ & $\begin{array}{c}\quad<10^{10.5} \\
>10^{10.5} \\
\text { all masses }\end{array}$ & $\begin{array}{l}-0.04 \pm 0.01(0.04) \\
-0.03 \pm 0.02(0.05) \\
-0.04 \pm 0.01(0.04)\end{array}$ & $\begin{array}{l}-0.21 \pm 0.07(0.18) \\
-0.26 \pm 0.10(0.27) \\
-0.23 \pm 0.07(0.21)\end{array}$ & $\begin{array}{l}-0.05 \pm 0.01(0.03) \\
-0.02 \pm 0.01(0.03) \\
-0.04 \pm 0.01(0.04)\end{array}$ & $\begin{array}{l}-0.24 \pm 0.04(0.13) \\
-0.15 \pm 0.07(0.16) \\
-0.21 \pm 0.04(0.15)\end{array}$ \\
\hline$z=0.4$ & $\begin{array}{c}\quad<10^{10.5} \\
>10^{10.5} \\
\text { all masses }\end{array}$ & $\begin{array}{l}-0.06 \pm 0.01(0.03) \\
-0.03 \pm 0.01(0.03) \\
-0.05 \pm 0.01(0.03)\end{array}$ & $\begin{array}{l}-0.28 \pm 0.04(0.14) \\
-0.19 \pm 0.08(0.17) \\
-0.25 \pm 0.04(0.16)\end{array}$ & $\begin{array}{l}-0.06 \pm 0.01(0.02) \\
-0.02 \pm 0.01(0.02) \\
-0.05 \pm 0.01(0.03)\end{array}$ & $\begin{array}{l}-0.24 \pm 0.04(0.10) \\
-0.15 \pm 0.06(0.12) \\
-0.21 \pm 0.02(0.12)\end{array}$ \\
\hline$z=0.95$ & $\begin{array}{c}<10^{10.5} \\
>10^{10.5} \\
\text { all masses }\end{array}$ & $\begin{array}{l}-0.07 \pm 0.03(0.12) \\
-0.05 \pm 0.04(0.11) \\
-0.05 \pm 0.02(0.11)\end{array}$ & $\begin{array}{l}-0.23 \pm 0.09(0.31) \\
-0.15 \pm 0.10(0.25) \\
-0.19 \pm 0.06(0.29)\end{array}$ & $\begin{array}{l}-0.07 \pm 0.02(0.07) \\
-0.06 \pm 0.02(0.05) \\
-0.06 \pm 0.01(0.06)\end{array}$ & $\begin{array}{l}-0.22 \pm 0.02(0.14) \\
-0.22 \pm 0.07(0.14) \\
-0.22 \pm 0.02(0.14)\end{array}$ \\
\hline$z=1.3$ & $\begin{array}{c}<10^{10.5} \\
>10^{10.5} \\
\text { all masses } \\
\end{array}$ & $\begin{array}{c}-0.08 \pm 0.02(0.13) \\
- \\
-0.07 \pm 0.03(0.13)\end{array}$ & $\begin{array}{c}-0.25 \pm 0.08(0.30) \\
- \\
-0.21 \pm 0.08(0.31) \\
\end{array}$ & $\begin{array}{c}-0.10 \pm 0.03(0.08) \\
- \\
-0.08 \pm 0.03(0.08)\end{array}$ & $\begin{array}{c}-0.30 \pm 0.04(0.14) \\
- \\
-0.28 \pm 0.05(0.17) \\
\end{array}$ \\
\hline
\end{tabular}

Notes. Estimations in both radial intervals are shown: $[0.5,1.5] r_{\mathrm{eff}}$ and $[0.5,1.0] r_{\mathrm{eff}}$. Bootstrap errors and standard deviations (parentheses) are included. Two sets of results are given, where the gradients were measured in different radial ranges.

number of analysed discs decreases with increasing redshift. The shaded areas denote the standard deviations.

The calculations of the metallicity gradients are done in the range $[0.5,1.5] r_{\mathrm{eff}}{ }^{1}$ and $[0.5,1.0] r_{\mathrm{eff}}$, which is the same interval used by Tissera et al. $(2016 \mathrm{a}, \mathrm{b})$ for the analysis of the gas and stellar metallicity profiles at $z \sim 0$. The results obtained by calculating the metallicity gradients in these two radial intervals are in agreement with each other as can be seen from Table 1 . We also tested extended calculations to $[0.5,2.0] r_{\text {eff }}$. However, the latter encompasses the external regions of the discs, which introduce extra noise when the discs have breaks or nearby satellite

\footnotetext{
1 The $r_{\mathrm{eff}}$ is the half-mass radius of the disc component (i.e. excluding
} the bulge). galaxies, and so on (Varela et al., in prep.), or have lower numbers of stellar particles.

We can see that, at all redshifts, metallicity gradients become steeper with stellar mass, but only up to $\log M \sim 10.3$. For higher masses, there is an inversion of this trend, and metallicity gradients become flatter. This leads to a characteristic U-shape for the relation of the stellar metallicity gradient with the stellar mass. The same behaviour is found for the normalised gradients. The overall trend is not the expected one for a linear extrapolation of the relation for the high-mass galaxies (or vice-versa, for the extrapolation for the low-mass end).

The observational trends determined for galaxies in the CALIFA survey by Sánchez-Blázquez et al. (2014) are more comparable to estimations within $[0.5,1.5] r_{\text {eff }}$. To illustrate this, 


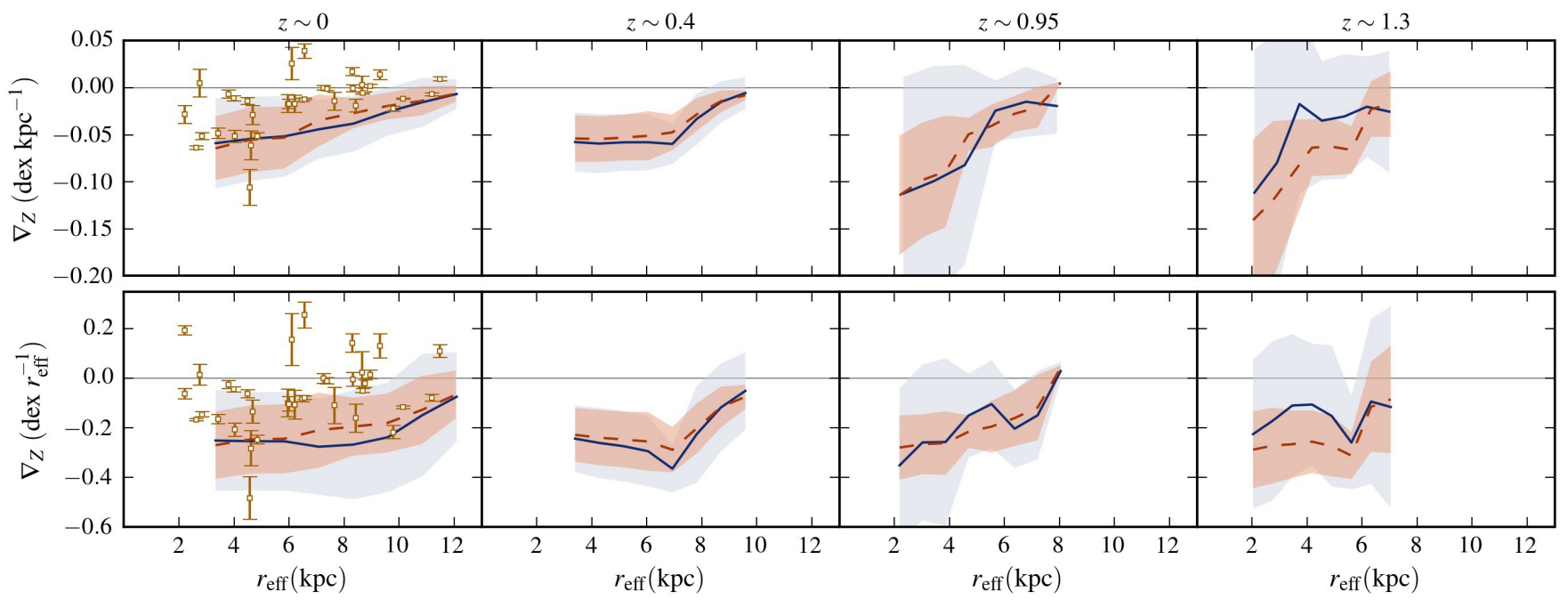

Fig. 3. Stellar metallicity gradients as a function of stellar $r_{\mathrm{eff}}$ for the analysed disc galaxies since $z \sim 1.3$ (upper panels). We also showed the distributions for the normalised stellar metallicity gradients at the same redshifts (lower panels). Standard deviations are included (shaded areas) as explained in Fig. 2.

in Fig. 2 we include the observations by Sánchez-Blázquez et al. (2014) and González Delgado et al. (2015) at $z \sim 0$. As reported by these papers, there is trend for a change in slopes so that lower-stellar-mass galaxies have flatter metallicity gradients than expected from a single linear relation. We note that a full agreement between the observed and the simulated normalised gradients is not expected since the scale-lengths and chemical abundances are estimated differently. The important point to highlight is the inversion of the metallicity trend with stellar mass in the observed and simulated relations at approximately the same stellar mass.

Previous works reported the existence of a correlation between gas-phase metallicity gradients and $r_{\text {eff }}$ (e.g. Prantzos \& Boissier 2000; Ho et al. 2015), which could be understood based on the hypothesis of angular momentum conservation of the gas as it flows in and settles into a disc structure. The correlation disappears when the gas-phase metallicity gradients are normalised by $r_{\text {eff }}$ for both observed and simulated discs (e.g. Sánchez et al. 2012; Ho et al. 2015; Tissera et al. 2016b). Within the standard model for disc formation (Fall \& Efstathiou 1980), the sizes of the discs are the result of angular momentum conservation. We understand this process as the net angular momentum conservation that can be the result of a balance between losses and gains along the evolutionary paths. Considering the relation between the specific angular momentum and the stellar mass reported for observed and simulated disc galaxies (e.g. Romanowsky \& Fall 2012; Fall \& Romanowsky 2013; Pedrosa et al. 2014; Genel et al. 2015; Teklu et al. 2015), the normalisation by the $r_{\mathrm{eff}}$ is expected to eliminate the massdependence, unless other processes act to strongly disturb the discs. Our results are in agreement with a scenario where gaseous discs form, conserving the angular momentum, overall, and the stellar populations form inside-out, enriching the ISM and producing metallicity gradients. These simulations take into account the assembly in a hierarchical scenario and thus, perturbations to the discs due to inflows, interactions, or mergers are considered, as well as the action of galactic outflows driven by SN feedback.

For the stellar metallicity gradients, Tissera et al. (2016a) reported a similar correlation at $z \sim 0$. We extended these

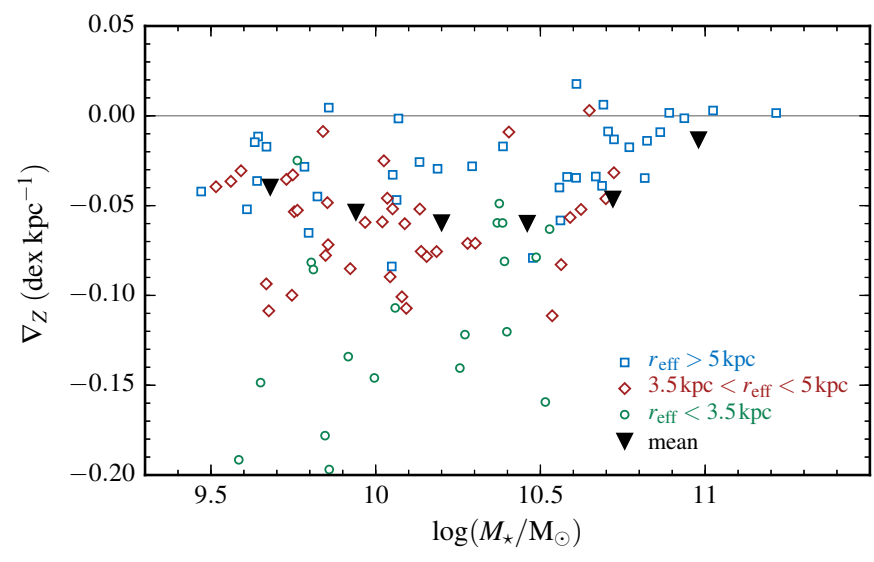

Fig. 4. Metallicity gradients as a function of stellar mass for all analysed redshifts. The colours denote different sizes of the discs $r_{\mathrm{eff}}>5 \mathrm{kpc}$ (blue stars), $3.5 \mathrm{kpc}<r_{\text {eff }}<5 \mathrm{kpc}$ (red stars) and $r_{\text {eff }}<3.5 \mathrm{kpc}$ (black stars). Mean gradients for the stacked distribution are shown (black triangles).

estimations to up to $z \sim 1$, finding similar trends as shown in Fig. 3. The Pearson correlation factors for these relations are $0.67,0.61,0.74$, and 0.65 for $z \sim 0,0.4,1$, and 1.3 , respectively. For the normalised gradients, the Pearson factors are 0.51, 0.34, 0.63 , and 0.44 , respectively. Although the correlation signals are less significant for the normalised metallicity gradients, they are high enough to suggest the non-existence of a common metallicity gradient for the SPs as the one found for the gas-phase metallicity gradients in observations (Sánchez et al. 2014) and simulations (Tissera et al. 2016a).

In order to gain further insight into the distribution of metallicity gradients as a function of stellar mass, we look for possible dependence on the global properties of the galaxies. Interestingly, the only clear trend is found with $r_{\text {eff }}$. In Fig. 4 we show all estimated metallicity gradients since $z \sim 1$ and the median values of the stacked distribution. At a given stellar mass, disc galaxies with flatter metallicity slopes have larger $r_{\text {eff }}$. Disc galaxies with the more negative stellar metallicity gradients tend to have the smaller $r_{\mathrm{eff}}$, on average. As can be seen, the inversion 
of the relation is recovered after the stacking of the four analysed redshifts. This suggests that the valleys of the distributions are located at approximately the same stellar mass, regardless of analysed redshift. We might then expect the physical process responsible for this behaviour to be independent of redshift.

In previous works, it has been shown that SN feedback is expected to be more efficient at driving galactic winds in smaller galaxies. Scannapieco et al. (2008) showed that the SN feedback model adopted in this work recovered such behaviour, producing a more efficient regulation of the star formation activity and driving stronger mass-loaded galactic winds in galaxies with shallower potential wells (see also de Rossi et al. 2010). As a consequence, disc components might be larger than expected since the gas can be heated up by SN feedback and blown out, acquiring angular momentum. Then it might cool down again contributing to build up the external parts of the discs where star formation can continue. This happens in some of the simulated discs within the lower stellar mass range. It is for these galaxies that our simulations produce gas-phase disc components and stellar discs with larger variety in metallicity gradients. Recent results on the stellar metallicity gradients from the MaNGA survey have been reported. Goddard et al. (2017) find most stellar metallicity gradients to be negative in agreement with previous works and to show an anticorrelation with the stellar mass. Using the MaNGA survey, Zheng et al. (2017) reported a correlation of the stellar metallicity gradients with stellar mass, albeit depending on galaxy morphology, in agreement with results from the CALIFA survey (Sánchez-Blázquez et al. 2014; González Delgado et al. 2015). Hence, observations do not yet provide a clear description of how the metallicity of the stellar populations depends on the stellar mass.

\subsection{The influence of radial migration}

Migration is a process that could also contribute to flatten the stellar metallicity gradients in the disc components of galaxies (e.g. Sellwood \& Binney 2002). Our simulations do not allow us to study this effect in detail as done by Grand et al. (2016), for example, because of the limited number of available snapshots and the numerical resolution. Nevertheless, we made a rough estimation of the radial displacement of star particles. Following Sánchez-Blázquez et al. (2009), in Fig. 5, we show the relation between the initial radius and the radial position at $z \sim 0$ for two disc galaxies with similar stellar masses and metallicity gradients (note that the initial radius corresponds to the first time the particle is identified as a star one). As can be seen there is variation in these correlations: one of them shows signs of having experienced stronger migration in the outer parts (upper panel) while the next one shows a larger displacement within the central regions (lower panel). For these galaxies, $\sim 22$ per cent of the stars within 1.5 of the optical radius show a displacement larger than 20 percent of the initial radius. Overall, we measured up to $\sim 20-25$ per cent variation in the positions within the $1.5 r_{\text {eff }}$ and the break radius (i.e. the radius where the stellar surface density showed a change of slope if a double power law is fitted). For this estimation, the analysis was done for the 16 most massive galaxies. This mass range goes down to $\sim 10^{10.5}$. In this mass interval, we did not find a clear trend with stellar mass, which could be associated to the inverse of the metallicity gradient relation (Fig. 2). Indeed, our galaxy sample does not cover the desired mass range with enough numerical resolution to evaluate this. Compared with the results of Sánchez-Blázquez et al. (2009) who found $\sim 40$ per cent displacement of stellar particles in the outskirts
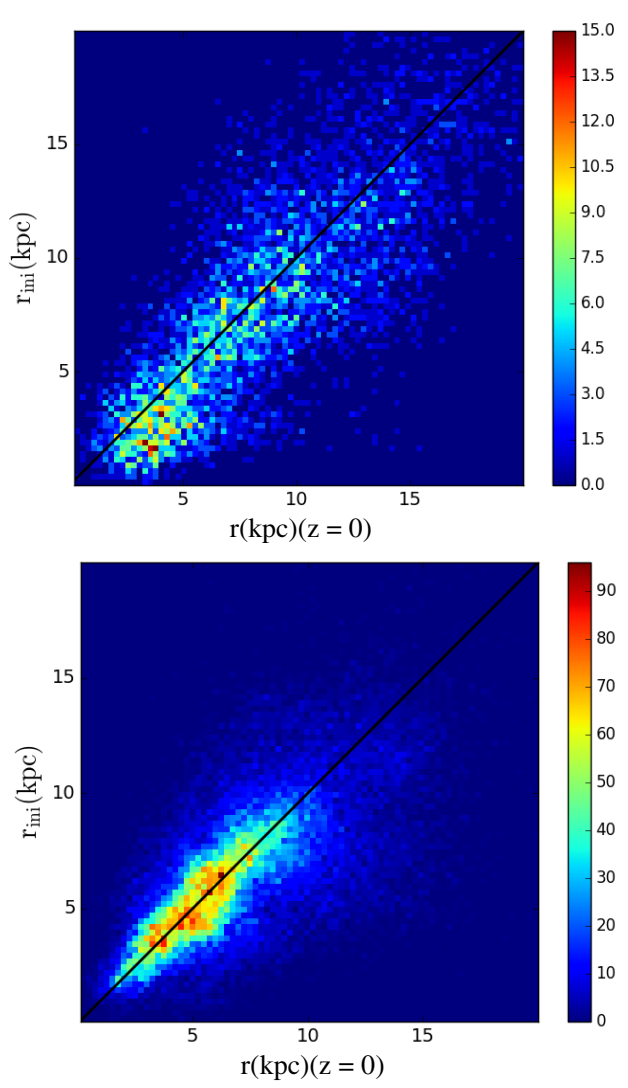

Fig. 5. Initial radius $\left(r_{\text {in }}\right)$ of stars (i.e. where it is first identified as a star) as a function of their radius $\left(r_{(z=0)}\right)$ at $z \sim 0$ for a galaxy with strong (upper panel) and weak (lower panel) signal of migration. Both galaxies have similar stellar metallicity gradients $\left(-0.02 \mathrm{dex} \mathrm{kpc}^{-1}\right.$ and $-0.03 \mathrm{dex} \mathrm{kpc}^{-1}$, respectively) and similar stellar masses $\left(1.1 \times 10^{10} M_{\odot}\right.$ and $4.7 \times 10^{10} M_{\odot}$, respectively).

of a disc galaxy, our simulated galaxies show weaker migration effects as mentioned above. We note that the SN feedback is different between the two models. Our numerical mass resolution is slightly better than that used by Sánchez-Blázquez et al. (2009) but Tissera et al. (2016b) have adopted a larger gravitational softening, which leads to a spatial resolution of $\sim 700 \mathrm{pc}$. However, Grand et al. (2016), where a more detailed analysis is performed, have higher resolution. Such a detailed analysis cannot be done with our simulations.

As an alternative assessment of the global impact of migration, we resort to the archeological estimation of the metallicity gradients using mono-age SPs identified at $z \sim 0$. The comparison of the actual metallicity gradients at a given redshift with the metallicity gradients of the SPs with similar age allows a global assessment of the effects of migration in our simulations. A larger diference between the evolution estimated by these two different methods would suggest an important effect of migration on our simulated metallicity profiles.

\subsection{The evolution of the stellar metallicity gradients}

In Fig. 6 (upper panel) we show the median metallicity gradients as a function of redshift, for the whole galaxy sample and for the high- and low-stellar-mass subsamples. We note that the median metallicity gradients of high-stellar-mass discs are systematically flatter than those of systems in the low-stellar-mass subsamples for $z<0.5$. This trend can be seen from Table 1 , where we summarise the metallicity gradients for their SPs as a function of stellar mass, for all analysed redshifts. However, as 

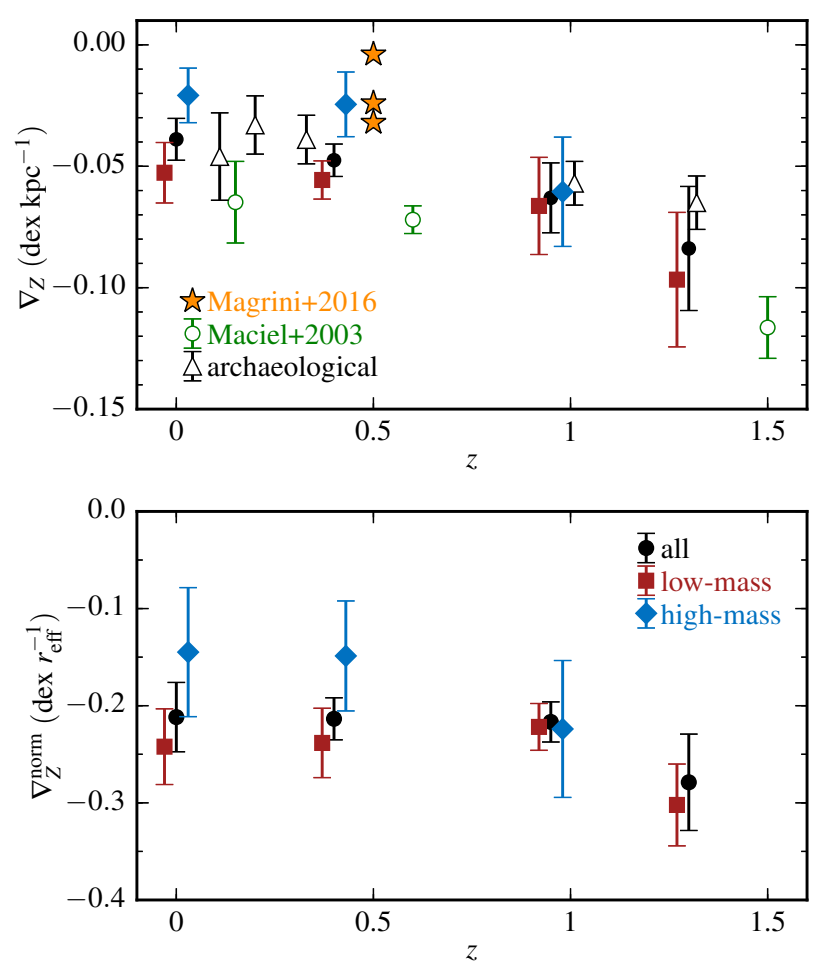

Fig. 6. Evolution of the stellar metallicity gradients. Upper panel: median slopes of the metallicity profiles for all simulated discs (black circles) and for those in the low-mass (red squares) and high-mass (blue diamonds) subsamples as a function of redshift. For comparison we include the observational estimations reported by Maciel et al. (2003) for the Milky Way (green open circles) and by Magrini et al. (2016; orange stars) for nearby galaxies. Estimations of the median metallicity gradients (open triangles) performed using the ages and spatial distributions of stars at $z \sim 0$ are included (see text for details). Lower panel: similar estimations for the normalised metallicity gradients. In both panels, the error bars are estimated using a bootstrap technique. For the sake of clarity, the simulation points are slightly offset horizontally in both panels.

our sample lacks massive galaxies at high redshift, it is difficult to distinguish between this effect and the actual change in the metallicity gradients with stellar mass as a function of redshift. Hence, hereafter, we focus on the global evolution of the metallicity gradients. Globally, we detect a mild evolution of the median metallicity gradients of the stellar discs in the analysed redshift interval as can be seen from Fig. 6 (upper panel) and Table 1. The mean change in slope is $-0.02 \pm 0.01 \mathrm{dex} \mathrm{kpc}-1$ from $z \sim 0$ to $z \sim 1$, which is also consistent with no evolution. We note that for these estimations we are using the stellar and metallicity distributions of the disc galaxies at each analysed redshift.

A different approach is to follow the archeological method of defining mono-age SPs at $z=0$ and estimating the metallicity gradients for each of them (e.g. Maciel et al. 2003; Magrini et al. 2016). From those metallicity gradients, it is possible to infer the level of evolution, assuming stars do not strongly modify their spatial locations after their birth. For the MW, Maciel et al. (2003) estimated a weak evolution consistent with the metallicity gradients to be more negative in the past. Recent estimations by Magrini et al. (2016) for galaxies in the Local Group suggest no evolution since $z \sim 0.5$. These authors could not determine if the lack of evolution was produced by a very effective SN feedback or by radial migration.
We apply the same method by defining mono-age SP adopting age intervals of 2 Gyr. In Fig. 6 (upper panel), we display these median gradients (open triangles), which are found to be in good agreement with those estimated using the actual distribution of the SPs in the discs at a given redshift (squares). The evolution derived from this method is $-0.03 \pm 0.02 \mathrm{dex} \mathrm{kpc}^{-1}$ over the whole redshift range analysed. For $z<0.5$, this analysis predicts no evolution of the stellar metallicity gradient, on average.

The archeological estimations encompass the effects of processes that can affect the distribution and dynamics of baryons such as galaxy interactions, strong bars, or migration. The fact that, on average, the evolution signals obtained from the stellar metallicity gradients of the stellar populations at a given redshift and from those derived by archeological means are in agreement supports a mild impact of these effects on the evolution of stellar metallicity gradients. As shown in this figure, our results are consistent with similar results reported for the MW stellar disc by Maciel et al. (2003, open circles) and those given by Magrini et al. (2016) as shown above. We note that these are global trends and as can be seen from Table 1 and Fig. 6, the dispersion is high enough to provide room for a diversity due to the different history of assembly of the galaxies in a hierarchical clustering scenario.

Our findings support the inside-out formation scenario (driven by angular momentum conservation) as the main process at work for spiral galaxies. However, this would not prevent other mechanisms from taking place and mixing up the SPs such as bars or migration. According to our results, they might be secondary effects which contribute to flattening the metallicity slopes and to increasing the dispersion depending on the history of formation of each galaxy (i.e. the formation of bars, subsequent gas accretion, or galaxy encounters).

\section{Conclusions}

We study the evolution of the metallicity gradients of the stellar discs in a hierarchical clustering scenario with the aim of analysing their evolution with time. The metallicity gradients reflect the enrichment of the ISM at the time of star formation and hence, can store information relevant to understanding the star formation process, the enrichment cycle, and the effects of galaxy assembly. Later, stars can be redistributed in the discs via dynamical processes such as galaxy-galaxy interactions or migration, altering the chemical patterns. Hence, the evolution of the stellar metallicity gradients holds important information for understanding galaxy formation.

We find a trend for our stellar discs to have metallicity gradients consistent with an inversion of the relation as a function of stellar mass. Our results show that some galaxies with stellar masses smaller than $\sim 10^{10.3} M_{\odot}$ have flatter stellar metallicity gradients than expected from a linear correlation. These galaxies are systematically found to have larger sizes than those with steeper negative metallicity slopes at a given stellar mass. As a consequence, a common metallicity gradient for the SPs as a function of stellar mass cannot be determined. SN feedback might be a major process determining the shape of the stellar metallicity versus stellar mass distributions, as it is known to be stronger in galaxies with shallower potential wells. The impact of the SN feedback on the metallicity distribution also depends on the history of assembly galaxies.

The metallicity gradients $\left(\mathrm{dex} \mathrm{kpc}^{-1}\right)$ correlate statistically better with $r_{\text {eff }}$. This relation also shows a clear increase of 
the dispersion for lower masses. The normalisation of the stellar metallicity gradient-size relation does not erase the correlation as is case for the gas-phase metallicity gradients (Tissera et al. 2016b). The action of dynamical effects could modify the metallicity gradients after the gas is transformed into stars. In the case of the gaseous discs, continuous gas infall can contribute to keeping such a relation in place, if it occurs with angular momentum conservation. Some of the simulated discs show evidence for migration; the estimated fractions are in the range $[10,25]$ per cent. These fractions have been robustly estimated for galaxies with stellar masses larger than $\sim 10^{10.3} M_{\odot}$. However, a similar analysis for smaller galaxies could not be done due to limited numerical resolution.

The stellar metallicity gradients are found to mildly evolve by $-0.02 \pm 0.01 \mathrm{dex} \mathrm{kpc}^{-1}$ from $z \sim 0$ to $z \sim 1$, albeit with a larger dispersion. This evolution has been obtained by using the stellar and metallicity distributions in each analysed disc at a given redshift. The normalisation of metallicity gradients by the corresponding $r_{\text {eff }}$ of each stellar disc component provides a median slope, which does not vary statistically up to $z \sim 1$. We note that there is a large dispersion at each analysed redshift, which can be ascribed to the variety of evolutionary paths. For $z>1$ we find indications of stronger evolution that should be constrained with a larger galaxy sample.

We also perform archaeological estimations of the metallicity gradients defining mono-age SPs. These yield an evolution of $-0.03 \pm 0.02 \mathrm{dex} \mathrm{kpc}^{-1}$, which is in agreement with that estimated from the actual distribution of stars. This finding supports a weak effect of stellar migration in our simulations, on average. We also point out that the level of evolution of the stellar metallicity gradients predicted by our simulations is consistent with those reported by recent observations that used the archeological approach (Maciel et al. 2003; Magrini et al. 2016).

Our results suggest that $\mathrm{SN}$ feedback is one mechanism responsible of producing more extended stellar discs with shallower metallicity gradients than expected for the extrapolation of the linear correlation with stellar mass. Migration could also explain the flattening of the metallicity gradients if it acts preferentially in some galaxies; for example, if they have experienced strong bar formation (Grand et al. 2016). Furthermore, galaxygalaxy interactions or small satellite accretions can also modify the metallicity profiles in the outer parts (e.g. Quillen et al. 2009; Perez et al. 2011). Disentangling the effects of these processes remains a challenge in a cosmological context.

Acknowledgements. We thank the anonymous referee for useful comments and suggestions. This work was partially supported by PICT 2011-0959 and PIP 2012-0396 (Mincyt, Argentina). P.B.T. acknowledges partial support from the Nucleo UNAB 2015 DI-677 of Universidad Andres Bello and Fondecyt 1150334 and the Southern Astrophysics Network (SAN) collaboration funded by Conicyt. R.E.G.M. acknowledges support from Ciência sem Fronteiras (CNPq, Brazil) P.S.B. acknowledges financial support from the: CONICYT-Chile Basal-CATA PFB-06/2007 and the AYA2013-48226-C3-1-P by the Ministerio de Ciencia e Innovacion. J.M.V. acknowledges financial support from AYA2013-47742-C4-1-P by the Spanish MINECO.

\section{References}

Barnes, J. E., \& Hernquist, L. 1996, ApJ, 471, 115

Brook, C. B., Kawata, D., Scannapieco, E., Martel, H., \& Gibson, B. K. 2007, ApJ, 661, 10

Bundy, K., Bershady, M. A., Law, D. R., et al. 2015, ApJ, 798, 7

Calura, F., Gibson, B. K., Michel-Dansac, L., et al. 2012, MNRAS, 427, 1401

Chiappini, C., Matteucci, F., \& Romano, D. 2001, ApJ, 554, 1044

Cresci, G., Mannucci, F., Maiolino, R., et al. 2010, Nature, 467, 811

de Rossi, M. E., Tissera, P. B., \& Pedrosa, S. E. 2010, A\&A, 519, A89

Di Matteo, P., Pipino, A., Lehnert, M. D., Combes, F., \& Semelin, B. 2009, A\&A, 499, 427
Di Matteo, P., Haywood, M., Combes, F., Semelin, B., \& Snaith, O. N. 2013, A\&A, 553, A102

Ellison, S. L., Patton, D. R., Simard, L., \& McConnachie, A. W. 2008, AJ, 135, 1877

Fall, S. M., \& Efstathiou, G. 1980, MNRAS, 193, 189

Fall, S. M., \& Romanowsky, A. J. 2013, ApJ, 769, L26

Genel, S., Fall, S. M., Hernquist, L., et al. 2015, ApJ, 804, L40

Gibson, B. K., Pilkington, K., Brook, C. B., Stinson, G. S., \& Bailin, J. 2013, A\&A, 554, A47

Goddard, D., Thomas, D., Maraston, C., et al. 2017, MNRAS, 465, 688

González Delgado, R. M., García-Benito, R., Pérez, E., et al. 2015, A\&A, 581, A103

Grand, R. J. J., Springel, V., Kawata, D., et al. 2016, MNRAS, 460, L94

Henry, R. B. C., Kwitter, K. B., Jaskot, A. E., et al. 2010, ApJ, 724, 748

Ho, I.-T., Kudritzki, R.-P., Kewley, L. J., et al. 2015, MNRAS, 448, 2030

Iwamoto, K., Brachwitz, F., Nomoto, K., et al. 1999, ApJS, 125, 439

Jones, T., Wang, X., Schmidt, K. B., et al. 2015, AJ, 149, 107

Lambas, D. G., Tissera, P. B., Alonso, M. S., \& Coldwell, G. 2003, MNRAS, 346, 1189

Li, C., Wang, E., Lin, L., et al. 2015, ApJ, 804, 125

Lia, C., Portinari, L., \& Carraro, G. 2002, MNRAS, 330, 821

Maciel, W. J., Costa, R. D. D., \& Uchida, M. M. M. 2003, A\&A, 397, 667

Magrini, L., Coccato, L., Stanghellini, L., Casasola, V., \& Galli, D. 2016, A\&A, 588, A91

Matteucci, F., \& Greggio, L. 1986, A\&A, 154, 279

Michel-Dansac, L., Lambas, D. G., Alonso, M. S., \& Tissera, P. 2008, MNRAS, 386, L82

Minchev, I., Chiappini, C., \& Martig, M. 2014, A\&A, 572, A92

Mollá, M., Ferrini, F., \& Díaz, A. I. 1997, ApJ, 475, 519

Mollá, M., Cavichia, O., Gavilán, M., \& Gibson, B. K. 2015, MNRAS, 451, 3693

Mosconi, M. B., Tissera, P. B., Lambas, D. G., \& Cora, S. A. 2001, MNRAS, 325, 34

Pedrosa, S. E., \& Tissera, P. B. 2015, A\&A, 584, A43

Pedrosa, S. E., Tissera, P. B., \& De Rossi, M. E. 2014, A\&A, 567, A47

Perez, M. J., Tissera, P. B., Scannapieco, C., Lambas, D. G., \& de Rossi, M. E. 2006, A\&A, 459, 361

Perez, J., Michel-Dansac, L., \& Tissera, P. B. 2011, MNRAS, 417, 580

Pilkington, K., Gibson, B. K., Brook, C. B., et al. 2012, MNRAS, 425, 969

Pipino, A., D'Ercole, A., \& Matteucci, F. 2008, A\&A, 484, 679

Prantzos, N., \& Boissier, S. 2000, MNRAS, 313, 338

Queyrel, J., Contini, T., Kissler-Patig, M., et al. 2012, A\&A, 539, A93

Quillen, A. C., Minchev, I., Bland-Hawthorn, J., \& Haywood, M. 2009, MNRAS, 397, 1599

Romanowsky, A. J., \& Fall, S. M. 2012, ApJS, 203, 17

Rupke, D. S. N., Kewley, L. J., \& Chien, L.-H. 2010, ApJ, 723, 1255

Sánchez, S. F., Kennicutt, R. C., Gil de Paz, A., et al. 2012, A\&A, 538, A8

Sánchez, S. F., Rosales-Ortega, F. F., Jungwiert, B., et al. 2013, A\&A, 554, A58

Sánchez, S. F., Rosales-Ortega, F. F., Iglesias-Páramo, J., et al. 2014, A\&A, 563, A49

Sánchez-Blázquez, P., Courty, S., Gibson, B. K., \& Brook, C. B. 2009, MNRAS, 398, 591

Sánchez-Blázquez, P., Rosales-Ortega, F. F., Méndez-Abreu, J., et al. 2014, A\&A, 570, A6

Scannapieco, C., Tissera, P. B., White, S. D. M., \& Springel, V. 2006, MNRAS, 371,1125

Scannapieco, C., Tissera, P. B., White, S. D. M., \& Springel, V. 2008, MNRAS, 389,1137

Sellwood, J. A., \& Binney, J. J. 2002, MNRAS, 336, 785

Springel, V. 2005, MNRAS, 364, 1105

Stanghellini, L., Magrini, L., Villaver, E., \& Galli, D. 2010, A\&A, 521, A3

Stott, J. P., Sobral, D., Swinbank, A. M., et al. 2014, MNRAS, 443, 2695

Teklu, A. F., Remus, R.-S., Dolag, K., et al. 2015, ApJ, 812, 29

Tissera, P. B. 2000, ApJ, 534, 636

Tissera, P. B., White, S. D. M., \& Scannapieco, C. 2012, MNRAS, 420, 255

Tissera, P. B., Machado, R. E. G., Sanchez-Blazquez, P., et al. 2016a, A\&A, 592, A93

Tissera, P. B., Pedrosa, S. E., Sillero, E., \& Vilchez, J. M. 2016b, MNRAS, 456, 2982

van der Wel, A., Franx, M., van Dokkum, P. G., et al. 2014, ApJ, 788, 28

Wiersma, R. P. C., Schaye, J., Theuns, T., Dalla Vecchia, C., \& Tornatore, L. 2009, MNRAS, 399, 574

Wilkinson, D. M., Maraston, C., Thomas, D., et al. 2015, MNRAS, 449, 328

Woosley, S. E., \& Weaver, T. A. 1995, ApJS, 101, 181

Yuan, T.-T., Kewley, L. J., Swinbank, A. M., Richard, J., \& Livermore, R. C. 2011, ApJ, 732, L14

Zaritsky, D., Kennicutt, Jr., R. C., \& Huchra, J. P. 1994, ApJ, 420, 87

Zheng, Z., Wang, H., Ge, J., et al. 2017, MNRAS, 465, 4572 